Revue des patrimoines

Le cheval et ses patrimoines (1ère partie)

\title{
Louis Desliens, un homme, un métier, une collection, un musée en devenir
}

Jean-Louis Coudrot

\section{OpenEdition}

\section{Journals}

Édition électronique

URL : http://journals.openedition.org/insitu/9648

DOI : $10.4000 /$ insitu.9648

ISSN : 1630-7305

Éditeur

Ministère de la Culture

Référence électronique

Jean-Louis Coudrot, "Louis Desliens, un homme, un métier, une collection, un musée en devenir », In Situ [En ligne], 18 | 2012, mis en ligne le 31 juillet 2012, consulté le 14 novembre 2019. URL : http:// journals.openedition.org/insitu/9648; DOI : 10.4000/insitu.9648

Ce document a été généré automatiquement le 14 novembre 2019.

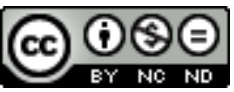

In Situ Revues des patrimoines est mis à disposition selon les termes de la licence Creative Commons Attribution - Pas d'Utilisation Commerciale - Pas de Modification 4.0 International. 


\title{
Louis Desliens, un homme, un métier, une collection, un musée en devenir
}

\author{
Jean-Louis Coudrot
}

1 Louis Desliens (1879-1975) est un vétérinaire originaire du département de l'Aube qui a exercé durant toute sa vie dans le Châtillonnais, région située en Côte d'Or, aux confins de la Bourgogne et de la Champagne (fig $\left.\mathbf{n}^{\circ} \mathbf{1}\right)$. Formé à l'école vétérinaire de MaisonsAlfort où il est admis en 1899, il en sort major de sa promotion en 1904 (fig. $\mathbf{n}^{\circ} \mathbf{2}$ ). Il s'installe comme vétérinaire praticien à Châtillon-sur-Seine en 1906 et partage son activité entre pratique et recherche. 


\section{Figure 1}

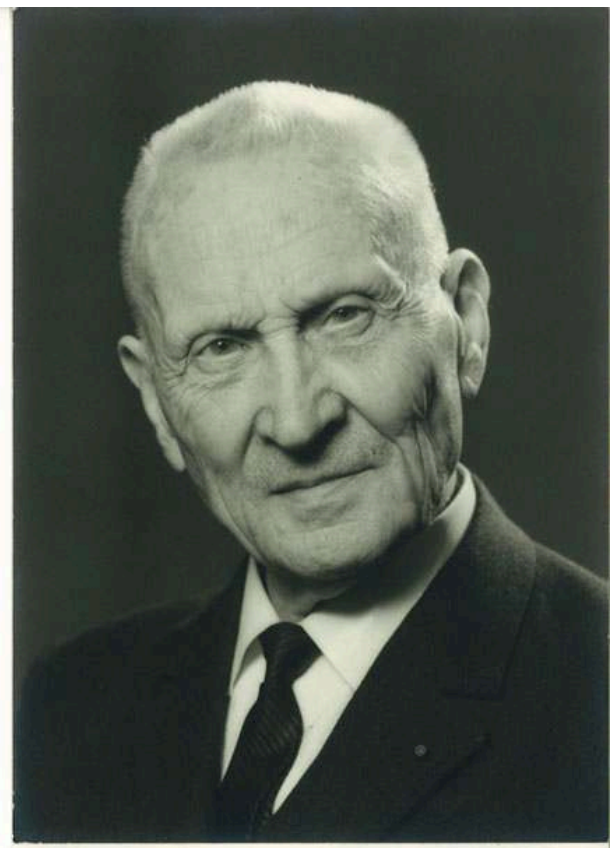

"La plus grande partie de ma vie professionnelle fut sans nuage,
accomplie tête baissée, avec la conviction que l'équité, la vérité, accomplie tête baissée, avec la conviction que l'équité, la vét

Louis DESLIENS
1879.1975

$$
\begin{aligned}
& 1879-1975 \\
& \text { Vétérinaire }
\end{aligned}
$$

Louis Desliens, 1879-1975.

(c) Coll. Desliens ; musée du Pays Châtillonnais-Trésor de Vix.

\section{Figure 2}

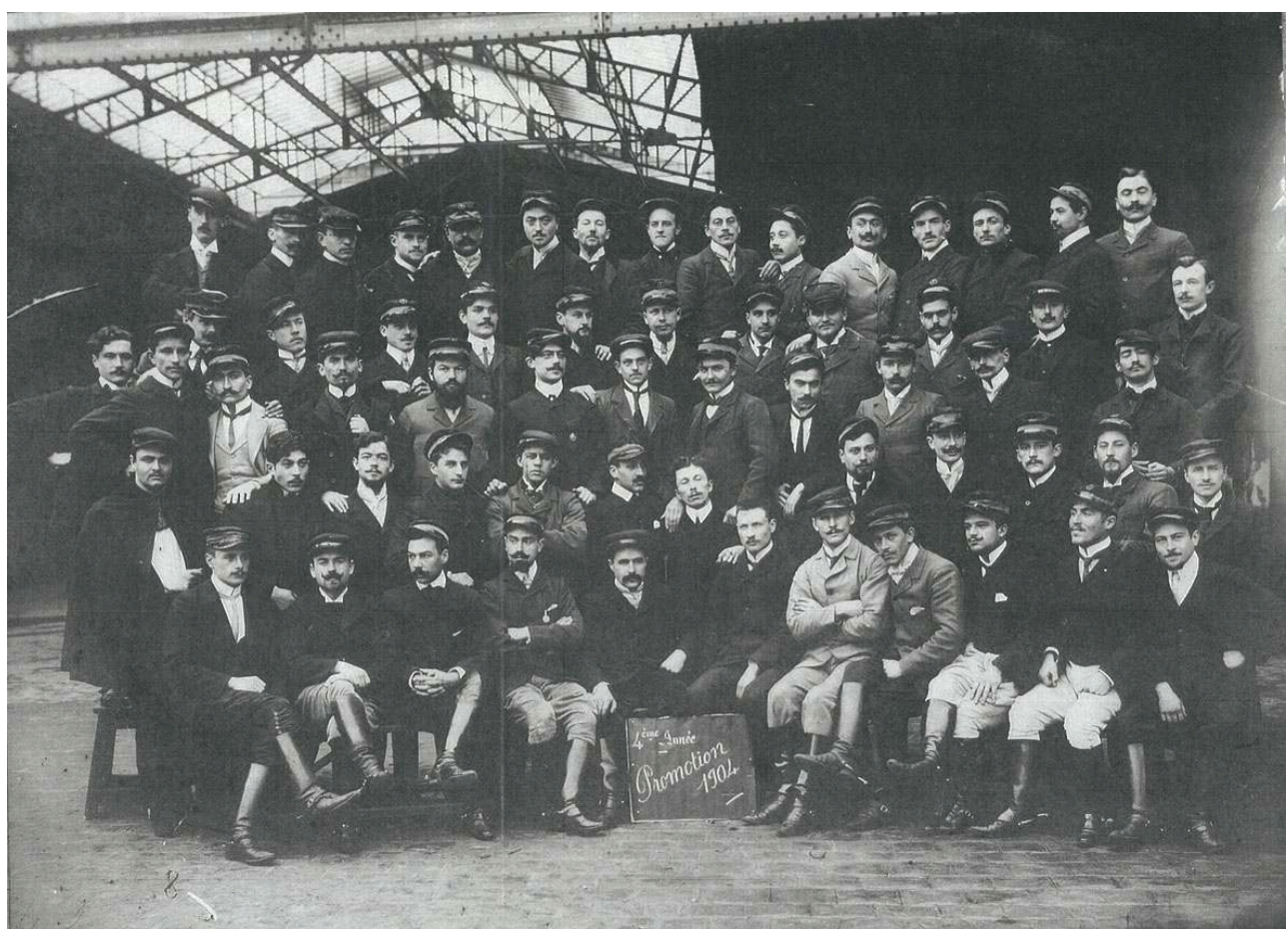

Louis Desliens, 4e année de la promotion 1904.

(c) Coll. Desliens ; musée du Pays Châtillonnais-Trésor de Vix. 
2 L'essentiel du travail de Louis Desliens, patron exigeant, est consacré à la médecine équine; il possède une écurie de six places où il hospitalise les chevaux (cryptorchiectomie, opération du javart). Ses interventions en urgences bovines sont plus rares car à l'époque, les cultivateurs hésitent à déranger le vétérinaire pour une vache : la seule urgence étant l'obstétrique. Sa renommée comme chirurgien l'appelle dans un rayon de $100 \mathrm{~km}$. Il accorde beaucoup d'importance à l'asepsie: pas de délivrance manuelle la veille d'une intervention ; en cryptorchiectomie, ses échecs sont à peine de $1 \%$. De 1903 , da te à laquelle il pratique sa première intervention chirurgicale à 1955 , où il met au point une nouvelle méthode de castration, il pratique la chirurgie.

Parallèlement à cette vie de praticien vétérinaire, il poursuit durant toute sa vie, des recherches sur la physiologie cardiaque et l'hémodynamométrie (fig. $\mathbf{n}^{\circ} \mathbf{3}$ ). Dès avant la première guerre (1906), il démontre l'innocuité du cathétérisme cardiaque en le pratiquant sur des chevaux et des bovins, expériences ignorées par Forssmann ${ }^{1}$ et Cournand $^{2}$. Sa première communication à l'Académie des Sciences date de 1916; il retraça l'histoire de 50 ans de recherche dans une conférence à Dijon, fort appréciée par le professeur Bressou, membre de l'Institut.

Figure 3

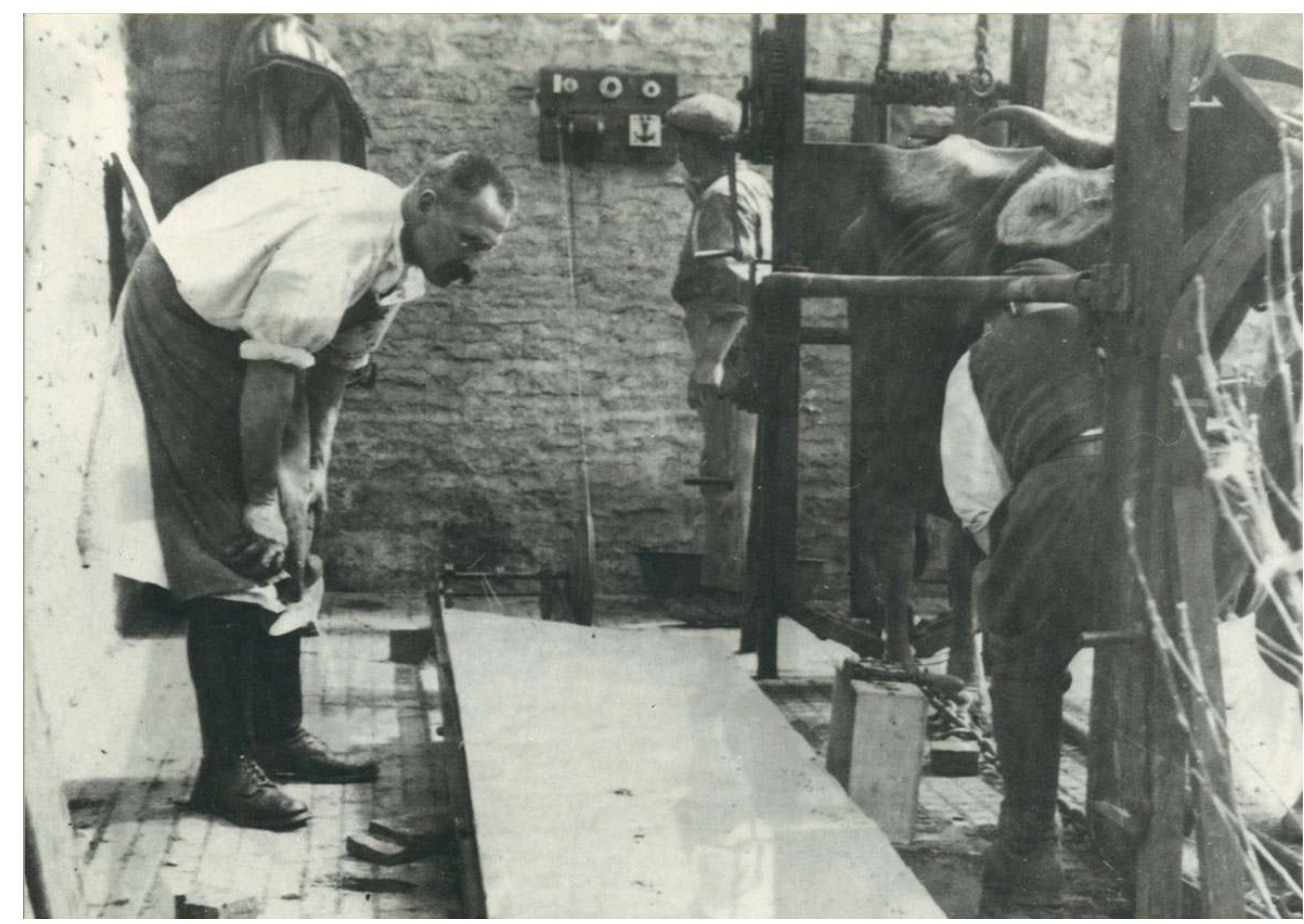

Enregistrement hémautographique.

(c) Coll. Desliens ; musée du Pays Châtillonnais-Trésor de Vix.

Louis Desliens fut le premier à utiliser la ponction artérielle transcutanée ainsi que les injections intra-artérielles pour le traitement du tétanos (13/11/1916). Ses expériences sur l'hématodynamométrie, mesure de la pression artérielle et veineuse, furent publiées dans un ouvrage paru dès 1935. Sa méthode directe permet d'approcher la pression sanguine de base chez le cheval qui reste indifférent aux ponctions artérielles (fig. $\left.\mathbf{n}^{\circ} 4\right)$. 
Figure 4

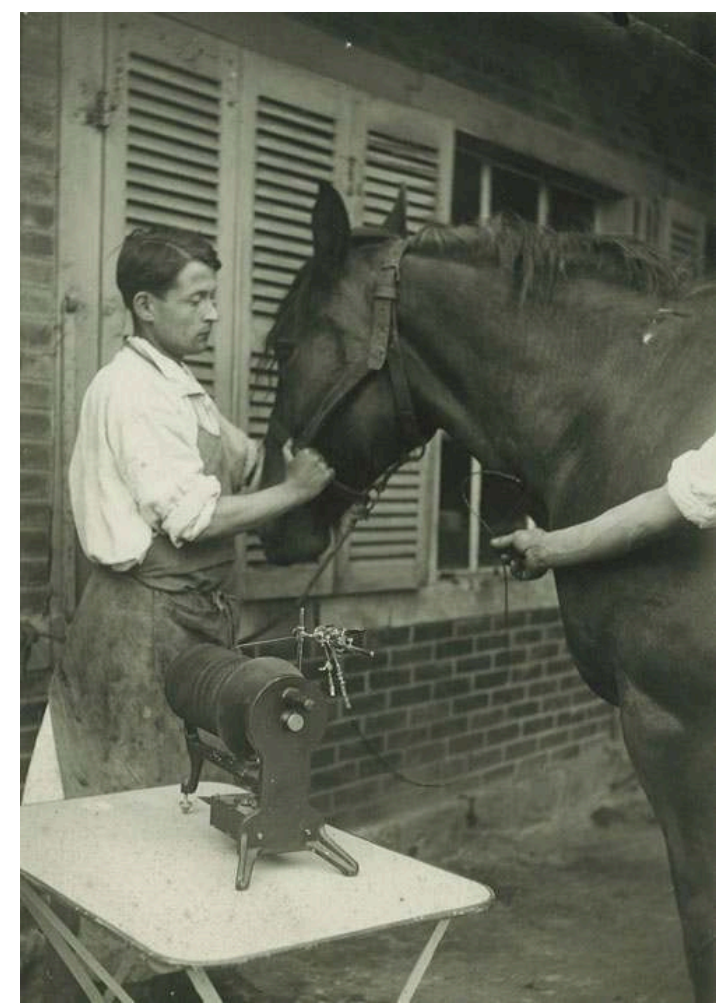

Prise de sang sur un cheval.

(c) Coll. Desliens; musée du Pays Châtillonnais-Trésor de Vix.

5 La transfusion sanguine fut également au centre du travail de recherche de ce praticien rural. Dès 1913, il procéda à la transfusion directe de la jugulaire du donneur au receveur; il utilisa ensuite une seringue de $500 \mathrm{ml}$; vers 1930, la citratation du sang lui permit de différer la transfusion. Cette technique fut utilisée pour le traitement des maladies infectieuses en transfusant du sang d'animaux convalescents.

6 Toute cette vie de praticien chercheur fut marquée par des publications et des notes d'expériences qui ont toutes été conservées par la famille Desliens (fig. $\mathbf{n}^{\circ} 5$ ). 


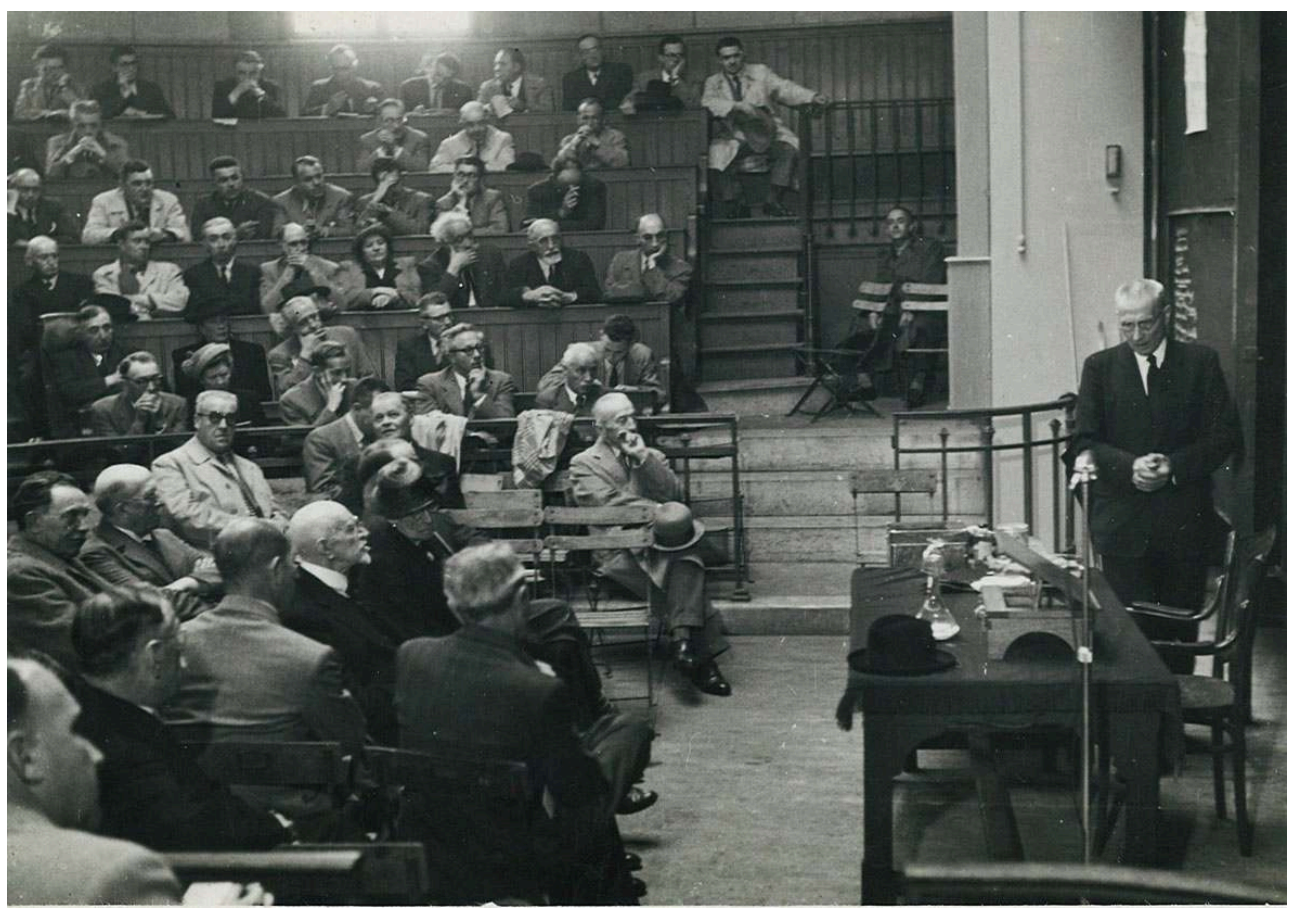

Assemblée dans un amphithéâtre vers 1960.

(c) Coll. Desliens ; musée du Pays Châtillonnais-Trésor de Vix.

7 Le matériel et les instruments conçus et réalisés par Louis Desliens constituent aujourd'hui une mémoire unique pour appréhender un aspect de la société sur plus d'un siècle autour de l'animal et au-delà sur la santé publique et la nourriture de l'homme.

8 Ainsi, toutes les collections, bases de l'activité de Louis Desliens, ont-elles été données par la famille Desliens ${ }^{3}$ au Musée du Pays Châtillonnais - Trésor de Vix où elles vont constituer une section originale unique en Europe, mais à bien considérer, un élément de continuité depuis la protohistoire dans cette région du Châtillonnais.

\section{Le programme scientifique et culturel du Musée du Pays Châtillonnais - Trésor de Vix}

9 Le Musée du Pays Châtillonnais - Trésor de Vix est situé à Châtillon-sur-Seine, agglomération de Côte d'Or, aux confins de la Bourgogne et de la Champagne, entre Troyes et Dijon. Depuis la protohistoire, sa position entre le bassin de la Seine et celui de la Saône et du Rhône a favorisé son développement et sa richesse. Pierre, fer, forêt, agriculture sont à l'origine d'une vitalité remarquable dont on retrouve la traduction dans les collections «encyclopédiques » jusqu'à l'époque moderne du musée. Il est l'animation d'une région qui dépasse les limites strictes de la géographie. Il permet de mettre en relations des territoires proches et lointains.

10 Le programme scientifique et culturel du musée, rédigé il y a près de dix ans, prévoit de présenter l'histoire de l'homme dans le Châtillonnais, un ensemble compact constitué par six cantons. Après une présentation géographique et géologique, le visiteur peut suivre l'impact de l'homme sur l'environnement et découvrir les moments forts de 
cette histoire, la période de l'Âge du fer, l'impact de Rome sur la région. L'emprise nouvelle de la forêt au Moyen Âge et ses fondations religieuses, le développement des nouvelles techniques, imprimerie, sidérurgie, industrie du drap et l'exploitation de la pierre et de la forêt. La dernière salle est consacrée aux gloires châtillonnaises du XIX ${ }^{\mathrm{e}}$ siècle, Nisard académicien et sénateur, Paul Louis Cailletet qui liquéfia l'air pour la première fois à Châtillon en 1877.

Figure 6

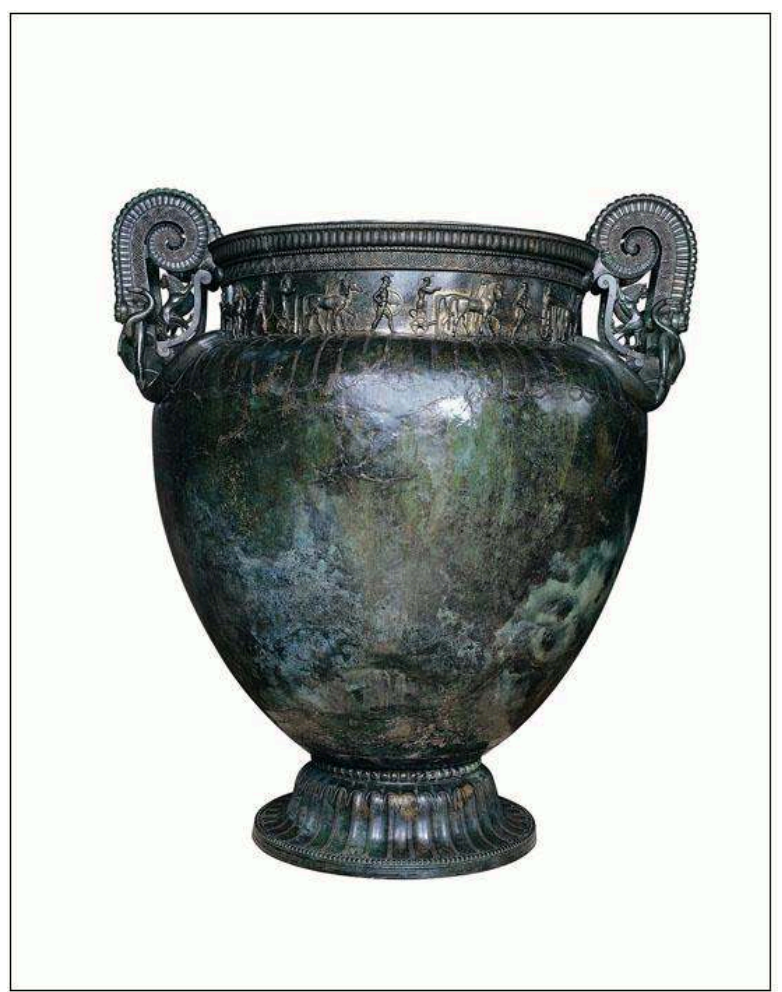

Cratère de Vix, 535 av. J.C., Grande Grèce.

Phot. Inv. J-L Duthu. (c) Musée du Pays Châtillonnais-Trésor de Vix.

Figure 7

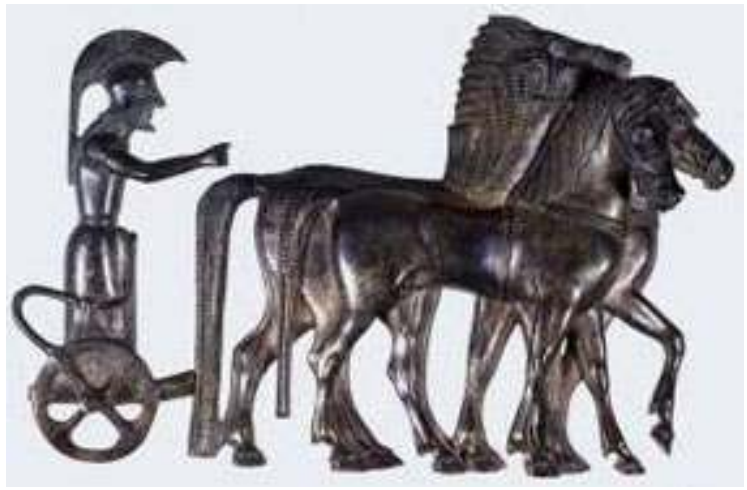

Cratère de Vix, quadrige détouré.

Phot. Inv. J-L Duthu. (c) Musée du Pays Châtillonnais-Trésor de Vix. 
11 La "Dame de Vix» et sa tombe exceptionnelle, véritable image du commerce international au $\mathrm{VI}^{\mathrm{e}}$ siècle avant notre ère, est une étape obligée pour mettre en relations les princes celtes du monde nord-alpin. Le cratère (fig. $\mathbf{n}^{\circ} \mathbf{6}$ ), vase grec en bronze, servant à mélanger le vin et l'eau pour le symposium est unique dans le monde antique avec son cortège de quadriges suivis d'hoplites (fig. $\left.\mathbf{n}^{\circ} \mathbf{7}\right)$. L'implantation galloromaine dans la partie orientale du territoire des Lingons a permis de constituer des collections originales : ex-voto des sanctuaires des eaux ou des temples consacrés aux divinités de l'abondance (Essarois, le Tremblois) avec de rares représentations de chevaux (fig. $\left.\mathbf{n}^{\circ} \mathbf{8}\right)$ (sculptures sur pierre ou fibules). Le site de Vertault (Vertillum) a permis la découverte de chevaux sacrifiés (fig. $\mathbf{n}^{\circ} \mathbf{9}$ ) et tous orientés de la même manière (le chanfrein est systématiquement fracassé) dans des fosses antérieures à l'époque gallo-romaine. L'étude de ces squelettes a permis la restitution de ces petits chevaux gaulois qui ne dépassaient pas 1,20 $\mathrm{m}$ au garrot (fig. $\left.\mathbf{n}^{\circ} \mathbf{1 0}\right)$.

Figure 8

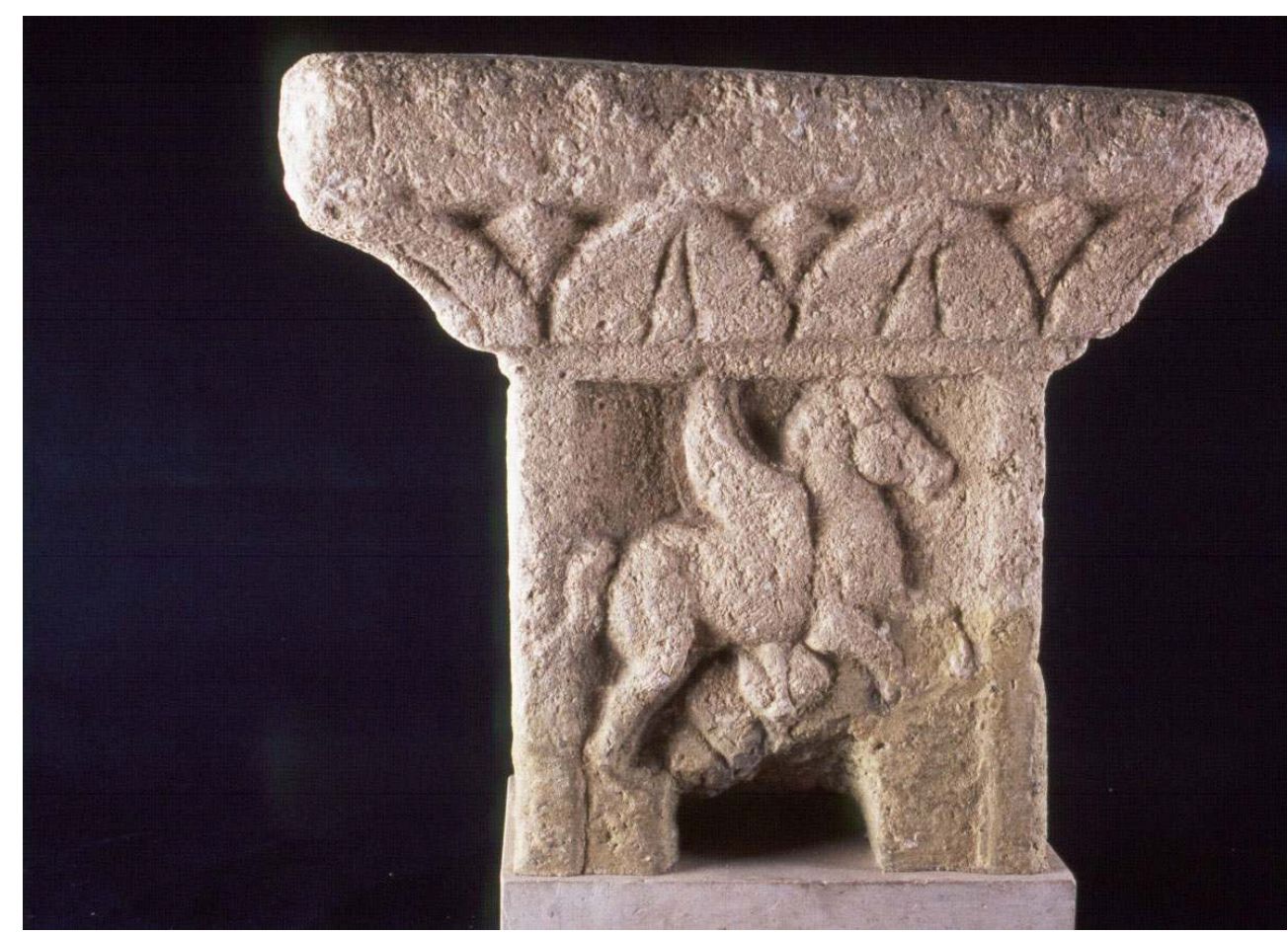

Vertault, Fontaine Pégase, époque gallo-romaine.

Phot. J-L Coudrot. (c) Musée du Pays Châtillonnais-Trésor de Vix. 
Figure 9

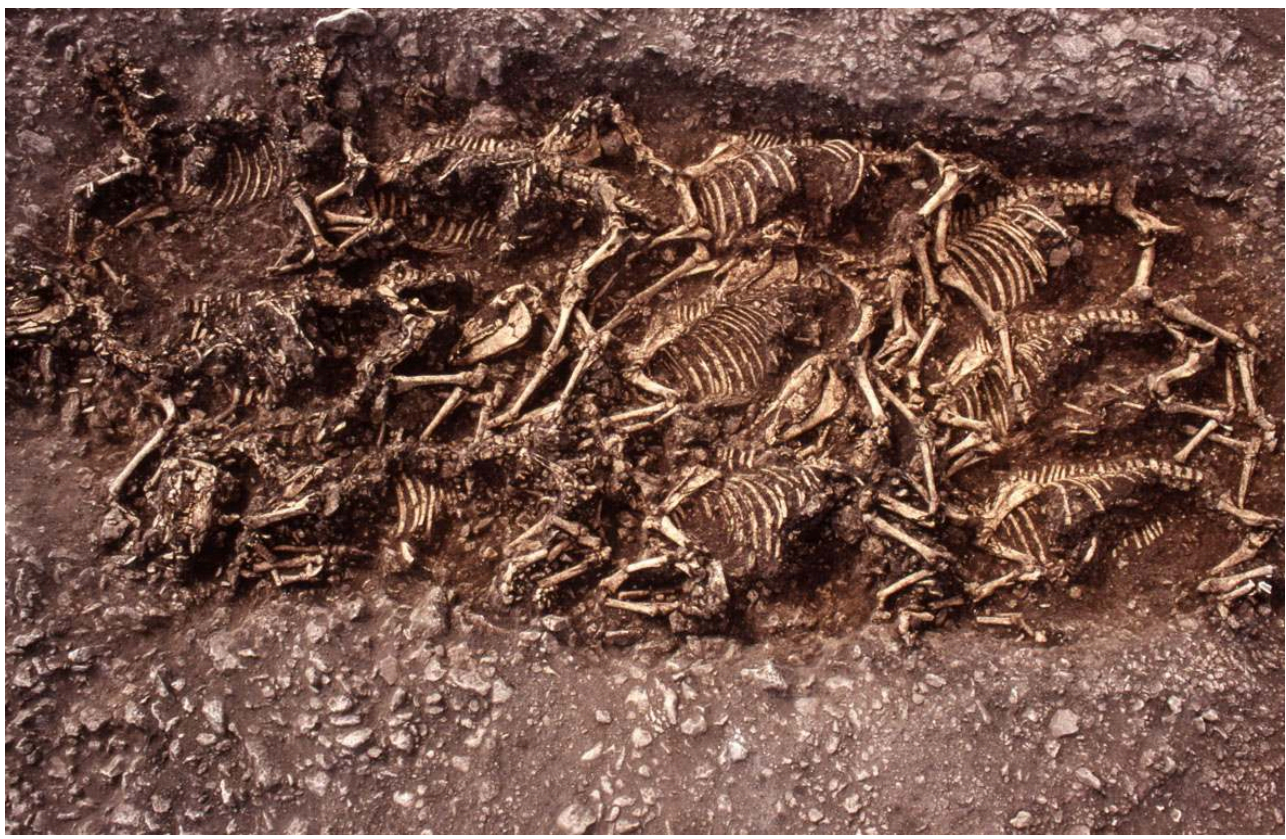

Vertault, ler siècle av. J. C., fosse aux chevaux.

Phot. J-P Garrault. @ Musée du Pays Châtillonnais-Trésor de Vix.

Figure 10

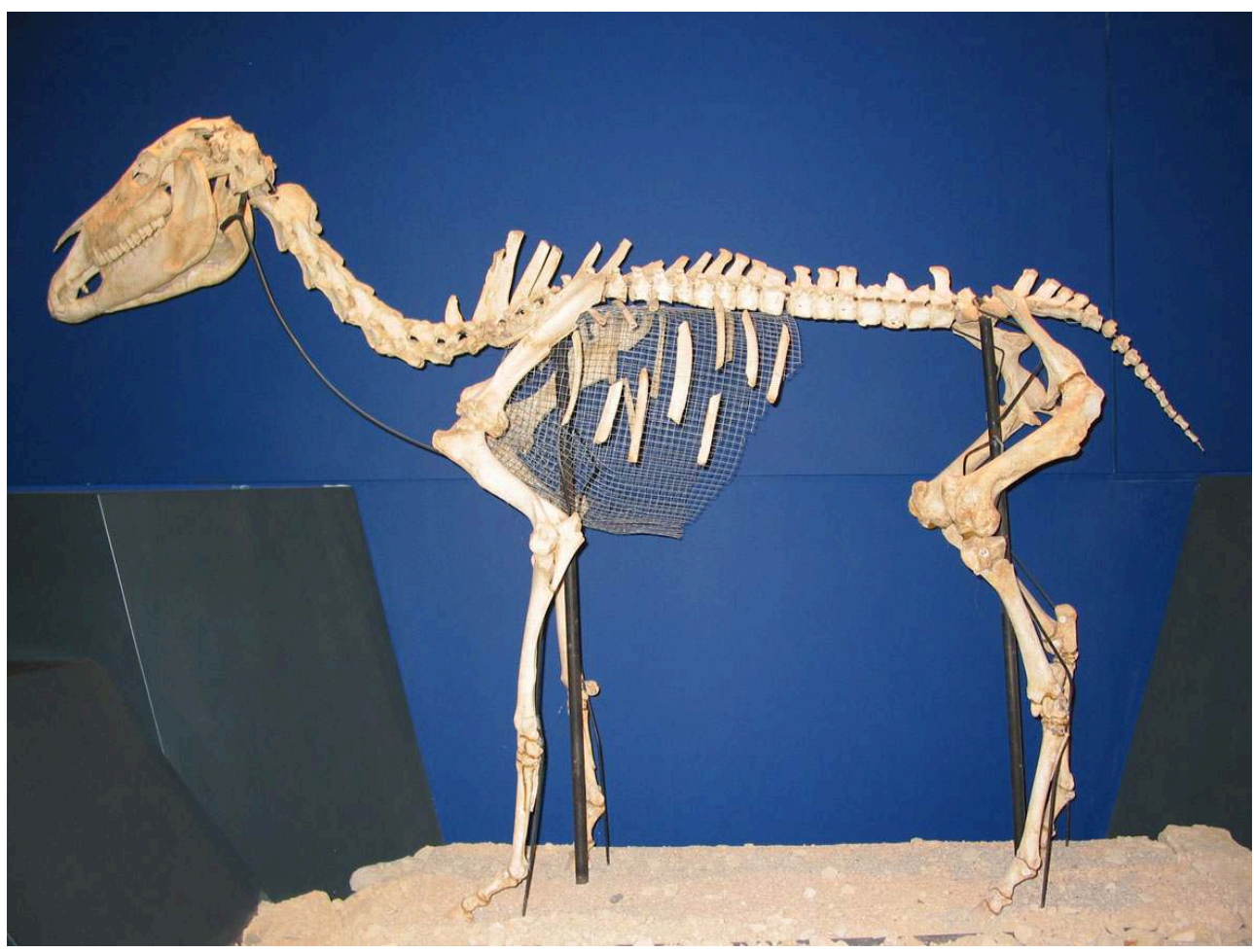

Vertault, ler siècle av. J. C.

Phot. J-L Coudrot. (c) Musée du Pays Châtillonnais-Trésor de Vix.

12 Le Moyen Âge, la Renaissance et l'époque moderne ne sont pas les périodes les moins actives dans le Châtillonnais. Des personnages importants marquent l'histoire et les 
collections: Robert de Molesme et Bernard de Clairvaux, mais aussi le Maréchal Marmont (fig. $\mathbf{n}^{\circ} \mathbf{1 1}$ ), duc de Raguse, figure essentielle de la région à l'origine du renouveau de la sidérurgie châtillonnaise et soutien majeur de l'agriculture et de l'élevage (mérinos).

Figure 11

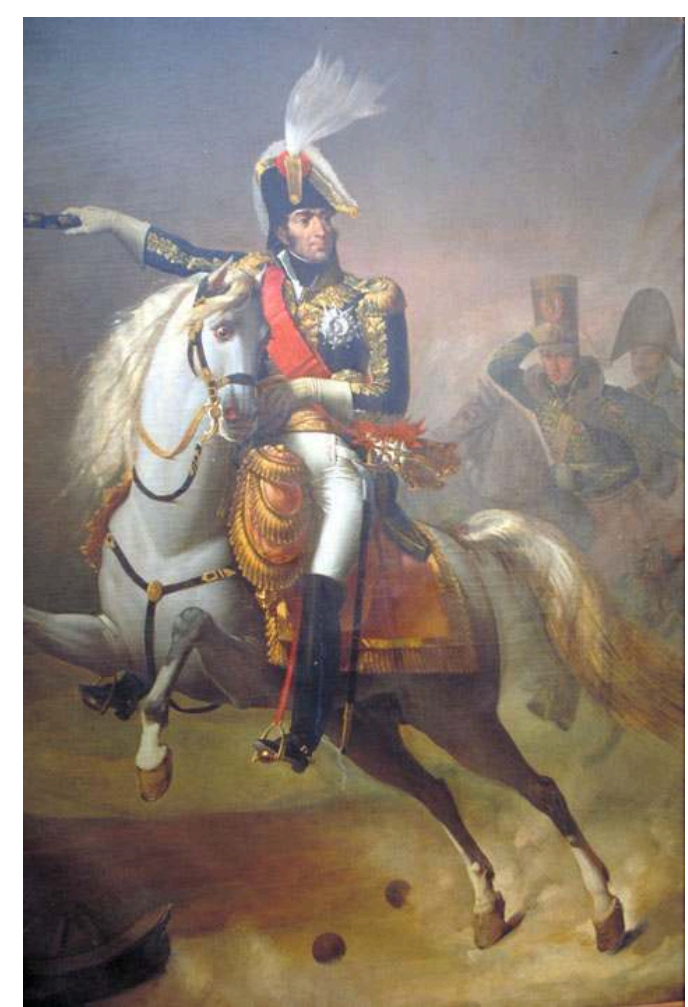

Maréchal Marmont, peinture de Barbier-Walbonne.

Phot. J-L Coudrot. (c) Musée du Pays Châtillonnais-Trésor de Vix.

Un dernier personnage présente un intérêt certain dans l'histoire de ce Châtillonnais : c'est Louis Desliens, un vétérinaire praticien et chercheur reconnu par ses pairs dont les importantes collections n'ont pu être présentées dans les salles des bâtiments conventuels de l'abbaye Notre-Dame.

Ainsi, tout visiteur spécialiste : archéologue, ethnologue, historien ou simple touriste peut découvrir les différents aspects de leur culture. Les habitants du Châtillonnais et plus généralement de la Bourgogne peuvent prendre conscience de leur patrimoine qui n'est pas encore totalement assimilé et compris.

\section{Le Musée Vétérinaire}

Dans le programme scientifique et culturel du musée du Pays Châtillonnais qui a reçu l'approbation de la direction des Musées de France en 1999, le transfert des collections du musée du Châtillonnais dans les bâtiments conventuels de l'abbaye Notre-Dame ne tenait pas compte des pièces de l'importante collection vétérinaire donnée par la famille Desliens, complétée depuis par des dons importants. Il a donc été proposé de les installer dans l'hôtel Philandrier (fig. $\mathbf{n}^{\circ} \mathbf{1 2}$ ) et son annexe. En effet, cet ensemble 
homogène peut être offert au public dans les étages de ces bâtiments, à moindre frais car les salles possèdent déjà une infrastructure muséographique (éclairage, alarme et vitrines).

Figure 12

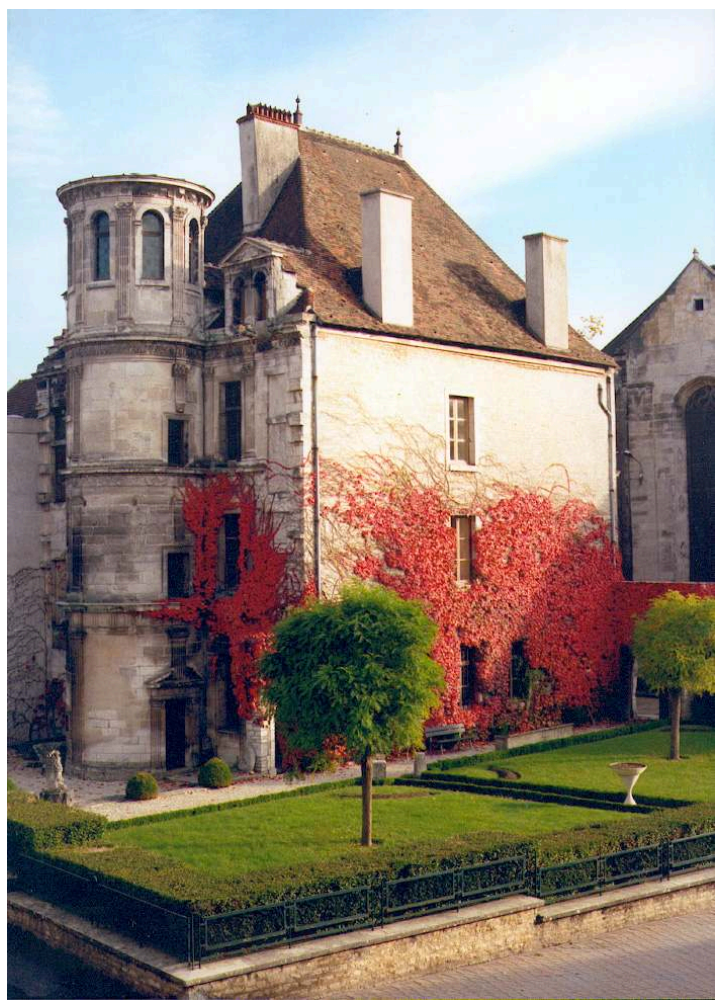

Hôtel Philandrier, XVIe siècle.

Phot. Ch. Labeaune. ( ) Musée du Pays Châtillonnais-Trésor de Vix.

Le rez-de-chaussée de cet immeuble, hôtel particulier du XVI siècle le plus remarquable de Châtillon-sur-Seine doit recevoir l'office de tourisme de la ville de Châtillon-sur-Seine dans des salles accueillantes à proximité de l'église Saint-Nicolas et de son parking.

Ainsi ce projet présente-t-il de nombreux avantages : de vastes locaux accessibles aux touristes arrivant à Châtillon-sur-Seine, plus faciles d'accès que le bureau actuel de l'office de tourisme, place Marmont. Il assure une surveillance du musée vétérinaire qui constitue un deuxième pôle muséographique à Châtillon-sur-Seine, incitant les touristes à circuler dans la ville et à découvrir ses monuments (Saint-Vorles, la Douix, Saint-Nicolas, Auditoire royal, vieux quartiers) et ses commerces.

Ce volet de musée vétérinaire, dont le thème sera unique en France, complète le projet du musée Fragonard situé dans l'Ecole vétérinaire d'Alfort, dont le thème est complémentaire du projet autour des collections du musée du Pays Châtillonnais, musée de l'animal domestique et de la médecine vétérinaire autour du cabinet d'anatomie du XVIII ${ }^{e}$ siècle organisé sur le thème des écorchés de Fragonard.

La présentation dans l'hôtel Philandrier est centrée sur l'histoire de l'art vétérinaire depuis les origines, de l'œuvre et des recherches de Louis Desliens (fig. $\mathbf{n}^{\circ}{ }^{\circ}$ ) au début du XXe siècle, époque de son installation comme praticien rural dont la notoriété dans 
la profession a largement dépassé les frontières et sur l'importance du rôle des vétérinaires dans la surveillance et le contrôle de l'alimentation contemporaine (vache folle, rage, ecchynoccose...) et la santé publique.

Figure 13

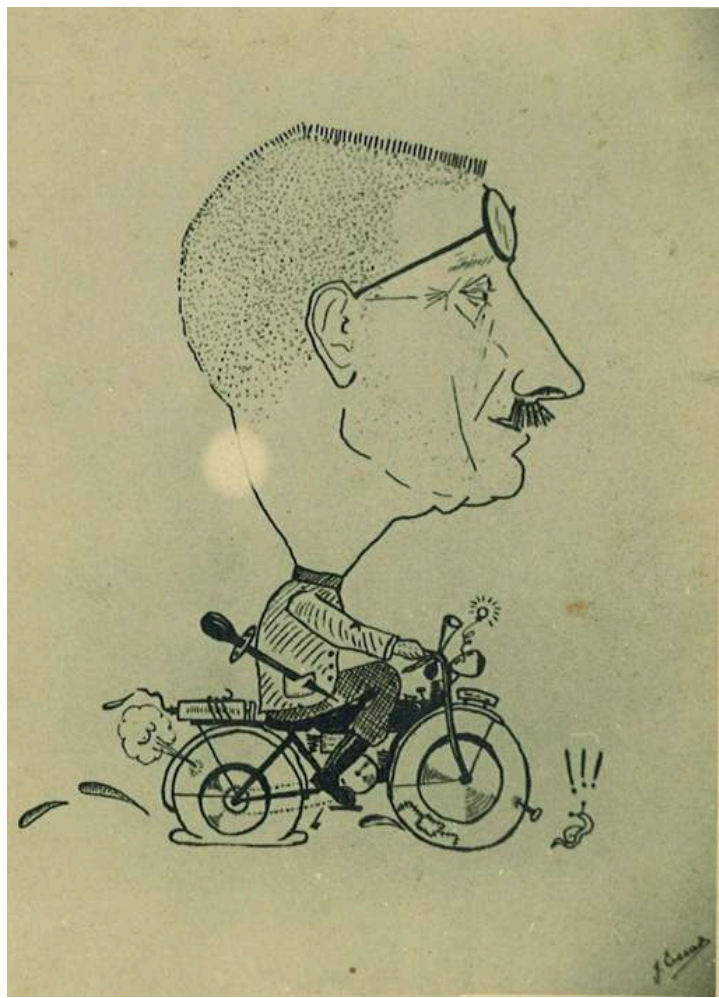

Louis Desliens, dessin, caricature.

(c) Coll. Desliens. Musée du Pays Châtillonnais-Trésor de Vix.

Les collections du Musée du Pays Châtillonnais forment un tout reconnu par les musées de France et dépendent naturellement des lois communes aux musées. De plus, la ville de Châtillon-sur-Seine s'était engagée moralement auprès des donateurs à réaliser, audelà d'une exposition temporaire, une présentation permanente des collections qui constituent un ensemble unique en Europe. Le seul exemple qui pourrait être comparable se trouve en Masurie à la frontière biélorusse et aujourd'hui dans un musée de Grande-Bretagne.

21 Le musée de la pratique vétérinaire sera la vitrine de la profession vétérinaire, pour le grand public, destinée à mieux la faire connaître et apprécier.

\section{Création d'un musée de la pratique vétérinaire et de la santé publique}

\section{Opportunité de la création d'un tel musée à Châtillon-sur-Seine}

L'activité vétérinaire trouve naturellement sa place dans un musée rural et il n'existe pas de véritable musée vétérinaire en France. 
l'École vétérinaire d'Alfort comporte des collections d'anatomie, d'anatomie-pathologie, de tératologie, de parasitologie, mais les objectifs du Musée du Pays Châtillonnais sont différents et complémentaires. Chaque école vétérinaire, chaque chaire possède du matériel ancien, mais on ne peut pas parler de véritable musée.

24

$$
\text { - en Pologne, un musée vétérinaire, }
$$

- en Grande-Bretagne, des expériences de musées vétérinaires au sein d'écomusées,

- en Allemagne, la collection du Dr Wentges de Münich qui se compose d'instruments vétérinaires et de médicaments anciens.

\section{Pourquoi un musée vétérinaire à Châtillon-sur-Seine ?}

La création d'un musée de la pratique vétérinaire dans cette région se justifie par le souvenir de Louis Desliens, praticien chercheur qui a vécu à Châtillon-sur-Seine de 1906 jusqu'à sa mort en 1975. Sa notoriété s'est étendue à toute la France, en particulier dans le domaine de la recherche en physiologie cardiaque sur les animaux et dans celui de la chirurgie du cheval et des bovins. Il a été précurseur de la cardiologie humaine moderne. Ce n'est pas un hasard s'il fut choisi pour représenter les praticiens vétérinaires français aux cérémonies du bicentenaire de l'École d'Alfort en 1965.

\section{Description du musée vétérinaire}

Ce musée pourra offrir au visiteur, d'une part ses collections permanentes, d'autre part des expositions temporaires, consacrées à des thèmes spécifiques. 


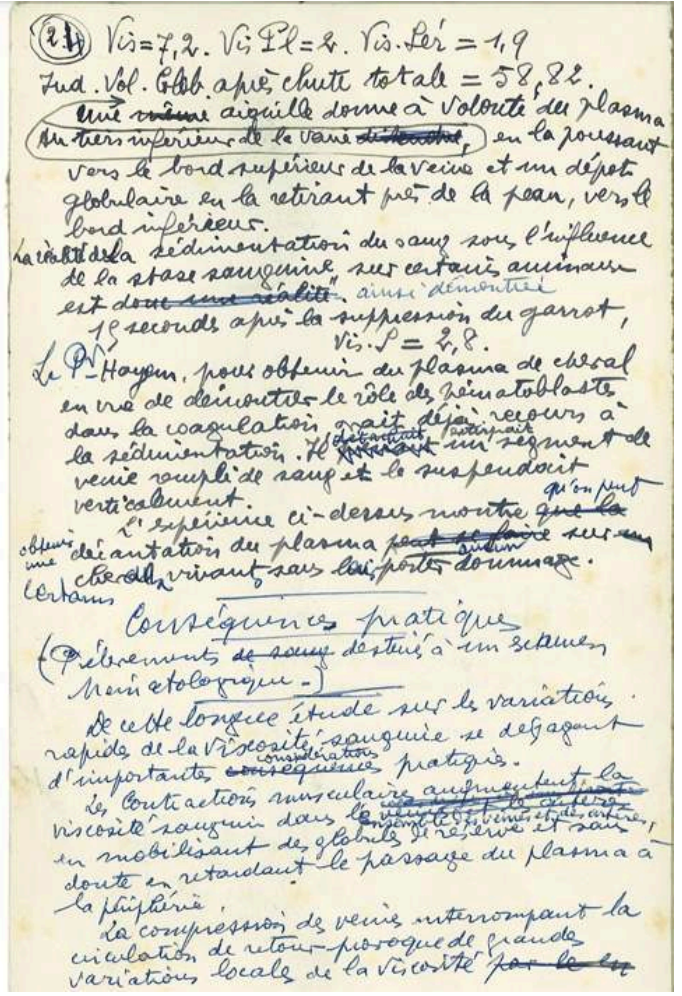

Note de travail 24.

(c) Coll. J-L Coudrot. Musée du Pays Châtillonnais-Trésor de Vix.

Le fonds permanent, constitué de l'héritage de Louis Desliens (fig. $\left.\mathbf{n}^{\circ} \mathbf{1 4}\right)$, de l'importante collection Crépin et de dons de vétérinaires et de laboratoires, compte :

- des instruments, dont certains illustrent des pratiques très anciennes (XVII ${ }^{e}$ siècle), - des médicaments anciens, quelques centaines d'échantillons, constituent une originalité rare qui permet de suivre à travers le $\mathrm{XIX}^{\mathrm{e}}$ et le $\mathrm{XX}^{\mathrm{e}}$ siècle, l'évolution de la pratique de la médecine vétérinaire (fig. $\mathbf{n}^{\circ} \mathbf{1 5}$ ),

- des pièces anatomiques (différentes parties de squelettes d'animaux, en particulier des têtes), anatomopathologiques (découvertes d'autopsie, c'est-à-dire calculs, bézoards, concrétions, etc...), ainsi que quelques pièces de tératologie, - des échantillons de parasites (arthropodes et helminthes) évoquant l'élevage et les maladies rencontrées chez les animaux domestiques de la région), - des catalogues et divers documents professionnels (quelques milliers de revues professionnelles dont certaines datent du début du $\mathrm{XX}^{\mathrm{e}}$ siècle, des correspondances de diverses sociétés savantes et de personnalités du monde vétérinaire ou médical, des annuaires vétérinaires) permettent de suivre l'évolution de la profession de vétérinaire. 
Figure 15

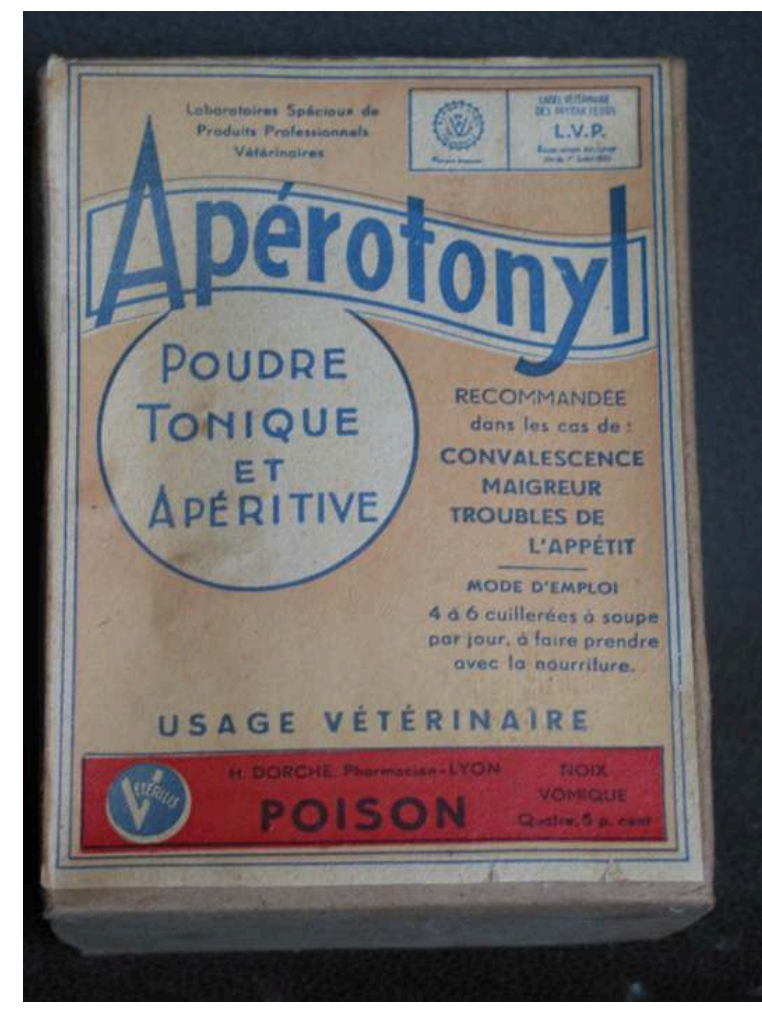

Médicament.

(c) Coll. J-L Coudrot. Musée du Pays Châtillonnais-Trésor de Vix.

C'est une bibliothèque composée de quelque 600 ouvrages de médecine vétérinaire et d'hippiatrie (dont certains datent du XVIII ${ }^{\mathrm{e}}$ siècle) et d'une centaine d'ouvrages de médecine ou de physiologie humaine qui sera à la disposition des chercheurs.

La reconstitution du bureau et du laboratoire de Louis Desliens, sera un point fort de cette présentation :

- ses cahiers d'expériences et ses agendas annuels de 1906 à 1974 relatant les interventions chirurgicales et les observations cliniques, permettant d'approfondir la pratique de chercheur,

- son matériel d'expérience (sondes, hémodynamomètres, trocarts, aiguilles, viscosimètres, hématimètre, cylindre enregistreur et tracés hémautographiques) rendant concrète son activité quotidienne (fig. $\mathbf{n}^{\mathbf{0}} \mathbf{1 6}$ ),

- des publications personnelles (ouvrages ou tirés-à-part d'articles) s'étalant sur soixante années,

- des photographies, des affiches, des prospectus,

- des médailles et quelques objets d'art. 
Figure 16

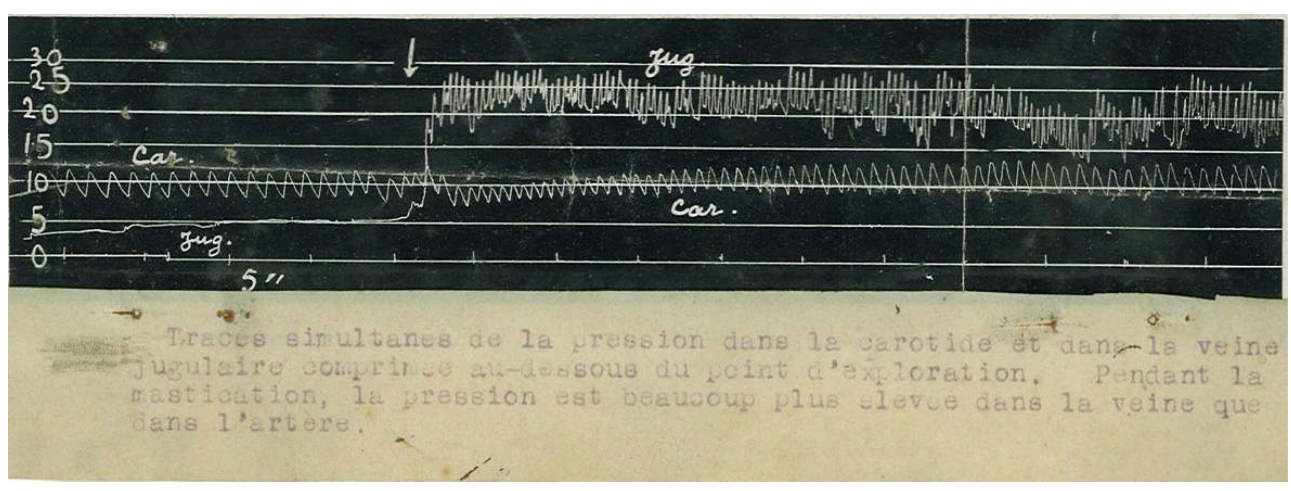

Relevé hémautographique.

(c) Coll. J-L Coudrot. Musée du Pays Châtillonnais-Trésor de Vix.

\section{Animation du musée}

\section{Le travail du vétérinaire, acteur du progrès scientifique}

30 Il est envisagé, pour des groupes spécialisés, une série d'expériences telles que Louis Desliens les réalisait, dans des lieux à définir, Lycée de La Barotte où l'activité du cheval est importante :

- ponction de la carotide du cheval,

- exploration des cavités cardiaques avec une sonde plastique et le manomètre,

- tracés hémautographiques dans le travail de Vinsot,

- démonstration, sur le cheval, de quelques interventions classiques d'autrefois

(couchage sur un lit de paille à l'aide de sept hommes, simulation de castration aux casseaux, simulation de la coupe de la queue).

\section{Des expositions temporaires}

31 Par exemple l'exposition de la collection du Dr Wentges serait une confrontation toutà-fait intéressante.

\section{Un centre de documentation}

32 Un grand nombre de documents (papier, microfiches, films) relatifs à l'histoire de la médecine vétérinaire et de sa pratique pourront être mis à la disposition d'étudiants et de chercheurs. L'accueil sera, bien entendu, organisé et permettra la réalisation d'études, thèses...

33 Par ailleurs, ce musée vétérinaire devra être en contact permanent avec les écoles vétérinaires, les instances régionales et nationales concernées, ainsi que les musées étrangers. Des liens étroits existent déjà avec le musée Fragonard et l'École vétérinaire de Maisons-Alfort.

34 Ce musée vétérinaire du Pays Châtillonnais sera un lieu de rencontre du monde vétérinaire qui pourra organiser colloques et séminaires avec les vétérinaires et l'industrie pharmaceutique. 


\section{BIBLIOGRAPHIE}

Bibliographie

DUGUET, Bénédicte. Louis DESLIENS vétérinaire praticien chercheur (ponctions artérielles, cathétérisme cardiaque, hémodynamométrie). Lyon : Université Claude Bernard, 1984.

DESLIENS, Louis. « Le javart cartilagineux (causes, traitements), nouveau procédé opératoire ». Revue Générale de Médecine Vétérinaire, 1918, 27, p. 297-315.

DESLIENS, Louis. « La recherche en médecine vétérinaire. Participation du praticien ». Bulletin de l'Académie Vétérinaire, 1964, 37, p. 368-389.

DESLIENS, Louis. Hémodynamométrie. Paris : Vigot Frères, 1935.

LEQUIME, J. « André Cournand, Prix Nobel de Médecine 1956 ». Revue Française d'Études cliniques et Biologiques, 1956, 1, p. 955-957.

LOOGEN, F. «In memory of Werner Forssmann ». Dans SNELLEN, (H.A), DUNNING, (A.J.) and ARNTZENIUS, (A.C.). History and perspectives of cardiology. Leiden : University Press, 1981, p. 39-43. ROUSSEAU, M. «À l'origine du cathétérisme cardiaque, deux oubliés : Chauveau (avec Marey) et Desliens ». La Nouvelle Presse Médicale, 1975, n³6, p. 3270-3272.

\section{NOTES}

1. - Werner Forssmann : Berlin 29/08/1904 - Schopfheim 01/06/1979.

2. - André F. Cournand : Paris 24/09/1895 - Great Barrington (États-Unis) 19/02/1988. Prix Nobel de physiologie ou médecine en 1956 avec Werner Forssmann "pour leurs découvertes concernant le cathétérisme cardiaque et des changements pathologiques dans le système circulatoire ".

3. - Michel Desliens : Châtillon-sur-Seine (Côte-d'Or) 14/09/1945 - Châtillon-sur-Seine (Côte-d'Or) 03/08/2001. Petit-fils de Louis Desliens, vétérinaire praticien à Châtillon, il a été durant toute sa vie celui qui a contribué à faire connaître l'œuvre de Louis Desliens. Il a constitué toute la collection donnée au Musée du Pays Châtillonnais - Trésor de Vix pour en faire une section autonome et une vitrine de la profession.

\section{RÉSUMÉS}

Louis Desliens (1879-1975), vétérinaire formé à Alfort, a consacré sa vie à la pratique vétérinaire mais également à la recherche. Son travail fut centré sur la médecine équine ; ses recherches sur l'hématodynamométrie furent publiées dès 1935 mais restèrent ignorées de Forssmann et Cournand, prix Nobel en 1956. Tout le matériel scientifique, les archives et les collections reflets de l'activité de Louis Desliens, ont été donnés par Michel Desliens, son petit-fils, au musée du Pays Châtillonnais - Trésor de Vix pour constituer un musée vétérinaire original unique en 
Europe en symbiose avec les collections du musée qui conservent des pièces exceptionnelles autour du cheval depuis la protohistoire : cratère grec en bronze avec défilé de char, chevaux gaulois sacrifiés de Vertault, outillage de maréchalerie du XVII ${ }^{\mathrm{e}}$ siècle... Le musée de la pratique vétérinaire Desliens sera une vitrine de la profession pour le grand public.

Louis Desliens ((1879-1975), a graduate of the Alfortschool of veterinary medicine, was a practicing veterinarian who devoted his life to research. His research, published as early as 1935, was focused on equine medicine, with a special interest in blood dynamometry. Some years later, it was adapted to human research by Forssmann and Cournand, who received the Nobel Prize in 1956 for their discoveries concerning heart catheterization and pathological changes in the circulatory system. Louis Desliens' grandson, Michel Desliens, donated the totality of his scientific material, his archives, and his collection to the Musée du Pays Châtillonnais - Trésor de Vix, for the purpose of creating a unique veterinary museum in Europe. The synergies between Desliens' legacy and the museum's existing collection are evident. Combined with such important pieces as a crater Greek bronze chariot with parade, sacrified Gallic horses by Vertault, and farrier tools of the 17th century, Desliens' collection promises to be a showcase for the veterinary profession that will have great appeal to the general public.

\section{INDEX}

Mots-clés : musée vétérinaire, Louis Desliens, Châtillon-sur-Seine, Vix, Vertault, Bourgogne, hématodynamométrie, crytochiectomie, cathéthérisme, Cournand

\section{AUTEUR}

\section{JEAN-LOUIS COUDROT}

Conservateur du Musée du Pays Châtillonnais - Trésor de Vix (jusqu'au 31 décembre 2011) jeanlouis.coudrot@laposte.net 